\title{
ON DISGUISED INVERTED WISHART DISTRIBUTION
}

\author{
A. K. GUPTA AND S. OFORI-NYARKO
}

(Communicated by Wei-Yin Loh)

\begin{abstract}
Let $A \sim W_{p}(n, \Sigma)$ and $A=Z Z^{\prime}$ where $Z$ is a lower triangular matrix with positive diagonal elements. Further, let $B=A^{-1}=W^{\prime} W$ have inverted Wishart distribution so that $W=Z^{-1}$. In this paper we derive the distribution of $M=W \Sigma W^{\prime}$. It is also shown that $\frac{n-p+1}{n p} T^{\prime} M T \sim F_{p, n-p+1}$ where $T \sim N_{p}\left(0, I_{p}\right)$ is independent of $M$.
\end{abstract}

\section{INTRODUCTION}

While deriving the minimax estimator of a normal covariance matrix when additional information is available on some coordinates [2], we were confronted with finding the distribution of a random variable of the type $\left(Z^{\prime} Z\right)^{-1}$ where $Z$ is a lower triangular matrix with positive diagonal elements such that $Z Z^{\prime}=$ $A(p \times p) \sim W_{p}(n, \Sigma)$. Here we first derive the distribution of $M=W \Sigma W^{\prime}$, where $W=Z^{-1}$. The distribution of $T^{\prime} M T$ is also derived where independently $T \sim N_{p}\left(0, I_{p}\right)$. We call $M$ a disguished inverted Wishart variable for reasons explained in $\S 3$. Tan and Guttman [3] derived the distribution of a disguised Wishart variable.

In $\S 2$ we present some preliminary results which are used in the sequel. In $\S 3$ main results of the paper are derived.

\section{SOME PRELIMINARY RESULTS}

The following lemmas are needed to derive the distribution of $M$. The proofs of Lemmas 1.1 and 1.2 are given in [1].

Lemma 2.1. If $M=M_{1} M_{1}^{\prime}$, where $M_{1}$ is a $p \times p$ lower triangular matrix with positive diagonal elements, then

$$
J\left(M \rightarrow M_{1}\right)=2^{p} \prod_{i=1}^{p} m_{i i(1)}^{p-i+1}
$$

where $m_{i i(1)}$ is the ith diagonal element of $M_{1}$. Also the transformation $N=$

Received by the editors July 7, 1993.

1991 Mathematics Subject Classification. Primary 62H10; Secondary 62H12.

Key words and phrases. Minimax estimation, risk, Jacobian, lower triangular matrix, $F$-distribution.

(C)1995 American Mathematical Society 
$M_{1}^{\prime} M_{1}$ gives

$$
J\left(N \rightarrow M_{1}\right)=2^{p} \prod_{i=1}^{p} m_{i i(1)}^{i} .
$$

Lemma 2.2. If $X$ and $A$ are $p \times p$ lower triangular matrices with positive diagonal elements, then the Jacobian of the transformation $Y=A X$ is

$$
J(Y \rightarrow X)=\prod_{i=1}^{p} a_{i i}^{i}
$$

and the $a_{i i}$ are the diagonal elements of $A$.

The following result is available in [3].

Lemma 2.3. Let $M^{[i]}=\left(m_{k l}\right), 1 \leq k, l \leq i$, be a submatrix of $M$. Then

$$
M=M_{1} M_{1}^{\prime}=\left[\begin{array}{ll}
M_{111} & 0 \\
M_{121} & M_{122}
\end{array}\right]\left[\begin{array}{cc}
M_{111}^{\prime} & M_{121}^{\prime} \\
0 & M_{122}^{\prime}
\end{array}\right]
$$

gives $M^{[i]}=M_{111} M_{111}^{\prime}$, with $M_{111}$ as an $i \times i$ principal diagonal block matrix, and $\left|M^{[i]}\right|=\prod_{j=1}^{i} m_{j j(1)}^{2}$. Also $\left|M^{[i-1]}\right|=\prod_{j=1}^{i-1} m_{j j(1)}^{2}$ and thus

$$
\left|M^{[i]}\right| /\left|M^{[i-1]}\right|=m_{i i(1)}^{2} \text {. }
$$

\section{Main Results}

We prove the main theorem using the three lemmas given in the previous section.

Theorem 3.1. Let $A$ be distributed as $W_{p}(n, \Sigma / n)$, where $\Sigma$ is a positive definite matrix of constants such that $\Sigma=Q Q^{\prime}$, where $Q$ is a lower triangular matrix. Then the distribution of $M=W \Sigma W^{\prime}$, such that $W^{\prime} W=A^{-1}$, is given by

$$
f(M)=C_{0}\left(\prod_{i=1}^{p} m_{i i(1)}^{2 i-p-1}\right)|M|^{-\frac{1}{2}(n+p+1)} e^{-\frac{n}{2} \operatorname{tr} M^{-1}},
$$

where $C_{0}=n^{n p / 2} / 2^{n p / 2} \Gamma_{p}(n / 2)$ and $m_{i i(1)}$ is given in (2.4).

Proof. If $A$ is distributed as $W_{p}(n, \Sigma / n)$, then $B=A^{-1}$ has the density

$$
f(B)=\frac{|\Sigma / n|^{-n / 2}|B|^{-\frac{1}{2}(n+p+1)} e^{-\frac{1}{2} \operatorname{tr} n \Sigma^{-1} B^{-1}}}{2^{n p / 2} \Gamma_{p}(n / 2)} .
$$

Let $B=W^{\prime} W$, where $W$ is lower triangular matrix. Then

$$
f(W)=\frac{|\Sigma / n|^{-n / 2}|B|^{-\frac{1}{2}(n+p+1)}\left(2^{p} \prod_{i=1}^{p} w_{i i}^{i}\right) e^{-\frac{1}{2} \operatorname{tr} n \Sigma^{-1}\left(W^{\prime} W\right)^{-1}}}{2^{n p / 2} \Gamma_{p}(n / 2)} .
$$

Since $\Sigma$ is positive definite, write $\Sigma=Q Q^{\prime}$ where $Q$ is lower triangular matrix. Then, using Lemma 2.1, we get

$$
\begin{aligned}
f(W)= & C_{0} 2^{p}\left|Q Q^{\prime}\right|^{-\frac{n}{2}}\left|W^{\prime} W\right|^{-\frac{1}{2}(n+p+1)} \\
& \cdot\left(\prod_{i=1}^{p} w_{i i}^{i}\right) \exp \left\{-\frac{1}{2} \operatorname{tr} n\left(Q Q^{\prime}\right)^{-1}\left(W^{\prime} W\right)^{-1}\right\} .
\end{aligned}
$$


Now let $M_{1}=W Q$. The Jacobian of this transformation is

$$
\left|\frac{\partial W}{\partial M_{1}}\right|=\prod_{i=1}^{p} q_{i i}^{-(p-i+1)},
$$

where $q_{i i}$ is the $i$ th diagonal element of $Q$. From (3.4) we obtain

$$
f\left(M_{1}\right)=C_{0} 2^{p}\left(\prod_{i=1}^{p} m_{i i(1)}^{i}\right)\left|M_{1} M_{1}^{\prime}\right|^{-\frac{1}{2}(n+p+1)} e^{-\frac{n}{2} \operatorname{tr}\left(M_{1} M_{1}^{\prime}\right)^{-1}},
$$

and because both $Q$ and $W$ are lower triangular matrices, $M_{1}$ also is a lower triangular matrix. Make the transformation

$$
M=M_{1} M_{1}^{\prime}=(W Q)(W Q)^{\prime}=W \Sigma W^{\prime} .
$$

From Lemma 2.1, the Jacobian of the transformation $M=M_{1} M_{1}^{\prime}$ is given by $2^{p} \prod_{i=1}^{p} m_{i i(1)}^{p-i+1}$, where $m_{i i(1)}$ is the $i$ th diagonal element of $M_{1}$ so that the distribution of $M$ is

$$
f(M)=C_{0}\left(\prod_{i=1}^{p} m_{i i(1)}^{2 i-p+1}\right)|M|^{-\frac{1}{2}(n+p+1)} \exp \left(-\frac{n}{2} \operatorname{tr} M^{-1}\right),
$$

which completes the proof.

The distribution (3.5) depends on the permutation of $M$. To illustrate this, let $p=2$. Then

$$
M=\left(\begin{array}{ll}
m_{11} & m_{12} \\
m_{12} & m_{22}
\end{array}\right)=M_{1} M_{1}^{\prime}=\left(\begin{array}{cc}
m_{11(1)} & 0 \\
m_{12(1)} & m_{22(1)}
\end{array}\right)\left(\begin{array}{cc}
m_{11(1)} & m_{12(1)} \\
0 & m_{22(1)}
\end{array}\right)
$$

so that

$$
m_{11}=m_{11(1)}^{2} ; \quad m_{12}=m_{11(1)} m_{12(1)} ; \quad m_{22}=m_{12(1)}^{2}+m_{22(1)}^{2} .
$$

Suppose one is interested in a permutation of $M$, say

$$
C=\left(\begin{array}{ll}
c_{11} & c_{12} \\
c_{12} & c_{22}
\end{array}\right)=\left(\begin{array}{ll}
m_{22} & m_{12} \\
m_{12} & m_{11}
\end{array}\right) .
$$

Then $C=\left(\begin{array}{ll}0 & 1 \\ 1 & 0\end{array}\right) M\left(\begin{array}{ll}0 & 1 \\ 1 & 0\end{array}\right)$. Writing $M=M_{1} M_{1}^{\prime}$,

$$
C=\left(\begin{array}{cc}
m_{12(1)}^{2}+m_{22(1)}^{2} & m_{11(1)} m_{12(1)} \\
m_{11(1)} m_{12(1)} & m_{11(1)}^{2}
\end{array}\right) \text {. }
$$

That is, $c_{11}=m_{12(1)}^{2}+m_{22(1)}^{2}, c_{12}=m_{11(1)} m_{12(1)}, c_{22}=m_{11(1)}^{2}$. Recall that

$$
c_{11}=m_{22}, \quad c_{12}=m_{12}, \quad c_{22}=m_{11} .
$$

We note that these are the same equations as in (3.6), except for appropriate changes in subscripts, and so nothing has really changed.

From (2.4), we have

$$
f\left(m_{11}, m_{12}, m_{22}\right)=C_{0}\left(m_{11} m_{22}-m_{12}^{2}\right)^{-\frac{n+2}{2}} m_{11}^{-1} \exp \left(-\frac{n}{2}\left(m^{11}+m^{22}\right)\right)
$$

where

$$
\left(\begin{array}{ll}
m^{11} & m^{12} \\
m^{12} & m^{22}
\end{array}\right)=\left(\begin{array}{ll}
m_{11} & m_{12} \\
m_{12} & m_{22}
\end{array}\right)^{-1}
$$


Similarly,

$$
g\left(c_{11}, c_{12}, c_{22}\right)=C_{0}\left(c_{11} c_{22}-c_{12}^{2}\right)^{-\frac{n+2}{2}} c_{22}^{-1} \exp \left(-\frac{n}{2}\left(c^{11}+c^{22}\right)\right) .
$$

An interesting observation is that if we make the transformation $N=M_{1}^{\prime} M_{1}$ (recall $\left.M=M_{1} M_{1}^{\prime}\right)$ with $\left|\partial N / \partial M_{1}\right|=2^{p} \prod_{i=1}^{p} m_{i i(1)}^{i}$,

$$
\left|M_{1}^{\prime} M_{1}\right|=\left|M_{1} M_{1}^{\prime}\right|, \quad \text { and } \operatorname{tr}\left(M_{1}^{\prime} M_{1}\right)^{-1}=\operatorname{tr}\left(M_{1} M_{1}^{\prime}\right)^{-1},
$$

then we find from (3.5) that

$$
f(N)=C_{0}|N|^{-\frac{1}{2}(n+p+1)} \exp \left(-\frac{n}{2} \operatorname{tr} N^{-1}\right) .
$$

That is, $N=M_{1}^{\prime} M_{1}=Q^{\prime} W^{\prime} W Q=Q^{\prime} B Q$ has the inverted Wishart distribution $W^{-1}\left(n, I_{p} / n\right)$. This is the reason why $M$ is called a disguised inverted Wishart variable. Tan and Guttman [3] derived the distribution of a disguised Wishart variable $R=P^{\prime} V P$ where $P P^{\prime}=\Sigma^{-1}$ and $\Sigma^{-1} \sim W_{p}\left(n-1, V^{-1} /(n-1)\right)$.

Motivated by the work of Tan and Guttman, we now derive the distribution of $G=T^{\prime} M T$ where $T \sim N_{p}\left(0, I_{p}\right)$ and is independent of $M$.

Theorem 3.2. Let $T \sim N_{p}\left(0, I_{p}\right)$ such that $T$ and $M$ are independent. Then the distribution of $G=T^{\prime} M T$ is such that

$$
G \sim \frac{n p}{n-p+1} F_{p, n-p+1}
$$

where $F_{p, n-p+1}$ denotes the $F$-distribution with $(p, n-p+1)$ degrees of freedom.

Proof. Write

$$
G=T^{\prime} M T=T^{\prime} M_{1} M_{1}^{\prime} T=T_{0}^{\prime} T_{0}
$$

where $T_{0}=M_{1}^{\prime} T$. Now conditional on $M_{1}$,

$$
f\left(T_{0} \mid M_{1}\right) \alpha\left|M_{1}\right|^{-1} \exp \left(-\frac{1}{2} \operatorname{tr} T_{0}^{\prime}\left(M_{1}^{\prime} M_{1}\right)^{-1} T_{0}\right) .
$$

Using (3.4) and (3.10) we have

$$
\begin{aligned}
& f\left(M_{1}, T_{0}\right) \alpha\left(\prod_{i=1}^{p} m_{i i(1)}\right)\left|M_{1}\right|^{-(n+p+2)} \\
& \cdot \exp \left(-\frac{1}{2} \operatorname{tr}\left[T_{0}^{\prime}\left(M_{1}^{\prime} M_{1}\right)^{-1} T_{0}+n\left(M_{1} M_{1}^{\prime}\right)^{-1}\right]\right),
\end{aligned}
$$

so

$$
\begin{aligned}
f\left(T_{0}\right) \alpha \int_{M_{1}} & \left(\prod_{i=1}^{p} m_{i i(1)}^{i}\right)\left|M_{1}\right|^{-(n+p+2)} \\
& \cdot \exp \left(-\frac{1}{2} \operatorname{tr}\left[T_{0}^{\prime}\left(M_{1}^{\prime} M_{1}\right)^{-1} T_{0}+n\left(M_{1} M_{1}^{\prime}\right)^{-1}\right]\right) d M_{1} .
\end{aligned}
$$

Now the exponent in the integrand of (3.11), apart from $(-1 / 2)$, may be written as

$$
\begin{aligned}
\operatorname{tr}\left[T_{0}^{\prime}\left(M_{1}^{\prime} M_{1}\right)^{-1} T_{0}+n\left(M_{1}^{\prime} M_{1}\right)^{-1}\right] & =\operatorname{tr}\left(M_{1}^{\prime}\right)^{-1}\left[T_{0} T_{0}^{\prime}+n I_{p}\right] M_{1}^{-1} \\
& =\operatorname{tr}\left(M_{1}^{\prime}\right)^{-1}\left(Q_{1}^{\prime} Q_{1}\right) M_{1}^{-1}
\end{aligned}
$$


where $Q=Q_{1}^{\prime} Q_{1}=T_{0} T_{0}^{\prime}+n I_{p}, Q_{1}$ is lower triangular, and $Q$ is positive definite. To verify this, note that for any $(p \times 1)$ vector $x \neq 0, x^{\prime} Q x=$ $\left(x^{\prime} T_{0}\right)^{2}+n x^{\prime} x>0$; hence, $Q$ is positive definite. Therefore, there exists a lower triangular $(p \times p)$ matrix $Q_{1}$ with positive diagonal elements such that $Q=Q_{1}^{\prime} Q_{1}$. Thus

$$
\begin{aligned}
\operatorname{tr}\left[T_{0}^{\prime}\left(M_{1}^{\prime} M_{1}\right)^{-1} T_{0}+n\left(M_{1}^{\prime} M_{1}\right)^{-1}\right] & =\operatorname{tr}\left(Q_{1} M_{1}^{-1}\right)^{\prime}\left(Q_{1} M_{1}^{-1}\right) \\
& =\operatorname{tr}\left[\left(M_{1} Q_{1}^{-1}\right)\right]^{\prime}\left(M_{1} Q^{-1}\right)^{-1}
\end{aligned}
$$

To evaluate the integral in (3.11), let $U=M_{1} Q_{1}^{-1}$. The Jacobian of the transformation is $\left|\partial U / \partial M_{1}\right|=\prod_{i=1}^{p} q_{i i}^{i-p-1}$. We can rewrite (3.11) as

$$
f\left(T_{0}\right) \alpha\left|Q_{1}\right|^{-(n+1)} \int_{U>0}\left(\prod_{i=1}^{p} u_{i i}^{i-p-1}\right)|U|^{-(n+1)} \exp \left(-\frac{1}{2} \operatorname{tr}\left(U U^{\prime}\right)^{-1}\right) d U
$$

The integral in (3.12) is constant. This is easily seen from (3.2) by replacing $n \Sigma^{-1}$ with $I_{p}$. Hence

$$
f\left(T_{0}\right) \alpha \frac{1}{\left|Q_{1}\right|^{n+1}}=\left|n I_{p}+T_{0} T_{0}^{\prime}\right|^{-((n+1) / 2)} .
$$

Using the fact that for a $(p \times p)$ matrix $M$,

$$
\int_{R^{p}}\left|M+t t^{\prime}\right|^{-m / 2} d t=\frac{\pi^{p / 2} \Gamma\left(\frac{m-p}{2}\right)}{\Gamma\left(\frac{m}{2}\right)|M|^{(m-1 / 2)}}
$$

and that $\int_{R^{p}} f\left(T_{0}\right) d T_{0}=1$, the density function of $T_{0}$ is given by

$$
f\left(T_{0}\right)=\frac{\Gamma\left(\frac{n+1}{2}\right)\left(1+\frac{T_{0}^{\prime} T_{0}}{n}\right)^{(n+1) / 2}}{(n \pi)^{p / 2} \Gamma\left(\frac{n+1-p}{2}\right)}
$$

and therefore $L=((n+1-p) / 2)^{1 / 2} T_{0}$ has a multivariate $t$-distribution with $(n+1-p)$ degrees of freedom. Making the transformation, $x_{i}=l_{i}^{2} /(n-p+1)$, it can be shown that $\prod_{i=1}^{p} x_{i}^{2}=L^{\prime} L /(n-p+1)$ is simply the inverted beta distribution $\beta^{\prime}(p / 2,(n+1-p) / 2)$ and hence has a $p F_{(p, n-p+1)} /(n-p+1)$ distribution. Therefore

i.e.,

$$
\frac{L^{\prime} L}{n-p+1} \sim \frac{p}{n-p+1} F_{p, n-p+1}
$$

so that $((n-p+1) / n) T_{0}^{\prime} T_{0} \sim p F_{p, n-p+1}$ or

$$
L^{\prime} L \sim p F_{p, n-p+1}
$$

$$
G=T_{0} T_{0}^{\prime}=T^{\prime} M T \sim \frac{n p}{n-p+1} F_{p, n-p+1} .
$$

\section{REFERENCES}

1. W. L. Deemer and I. Olkin, The Jacobians of certain matrix transformations useful in multivariate analysis, based on lectures of P. L. Hsu at the University of North Carolina, 1947; Biometrika 38 (1951), 345-367.

2. A. K. Gupta and S. Ofori-Nyarko, Improved minimax estimators of covariance and precision matrices when additional information is available on some coordinates, Department of Mathematics and Statistics, Bowling Green State University, Technical Report No. 93-11, 1993. 
3. W. Y. Tan and I. Guttman, A disguised Wishart variable and a related theorem, J. Roy. Statist. Soc. Ser. B 33 (1971), 147-152.

Department of Mathematics and Statistics, Bowling Green State University, BowlING GREEN, OHIO 43403

E-mail address: guptaCandy.bgsu.edu 